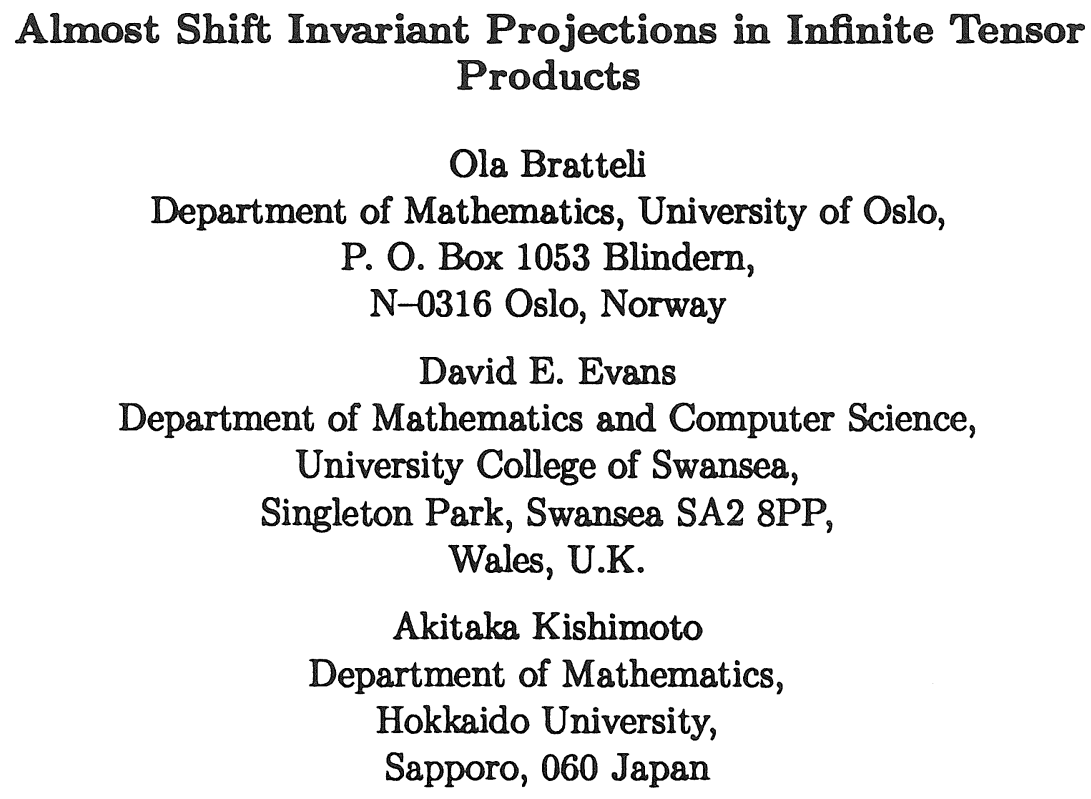

\title{
The Rohlin property
}

Let $\sigma$ be an automorphism of a unital $C^{*}$-algebra $A$. Following [Con], [HO], [BEK] we will say that the dynamical system $(A, \sigma)$ has the Rohlin property if there exists a strictly increasing sequence $\left(n_{k}\right)_{k}$ of positive integers such that for each $m=n_{k}$ there is a sequence

$$
\left(e_{i}^{(j)} \mid i=0,1, \ldots, m\right)_{j}
$$

of families of projections in $A$ with

$$
\begin{aligned}
& e_{m}^{(j)}=e_{0}^{(j)}, \\
& \sum_{i=0}^{m-1} e_{i}^{(j)}=1, \\
& \lim _{j \rightarrow \infty}\left\|\sigma\left(e_{i}^{(j)}\right)-e_{i+1}^{(j)}\right\|=0
\end{aligned}
$$

for $i=0,1, \ldots, m-1$, and

$$
\lim _{j \rightarrow \infty}\left\|\left[x, e_{i}^{(j)}\right]\right\|=0
$$

for all $x \in A, i=0,1, \ldots, m-1$.

It was established in [Voi, Lemma 3.3] (see also [BKRS], Lemma 2.1]) that if $A$ is a UHF algebra (or more generally, if $A$ is a unital AF algebra and $\sigma$ is an approximately inner automorphism) and $(A, \sigma)$ has the Rohlin property, then $\sigma$ is an almost inductive limit automorphism. This means 
that for any $B \in \mathcal{F}(A)=$ the set of finite dimensional "subalgebras of $A$ containing the unit of $A$, and any $\varepsilon>0$, there is a $C \in \mathcal{F}(A)$ such that $B \subseteq \subseteq^{\varepsilon} C$ and $d(\alpha(C), C)<\varepsilon$. Here we use the terminilogy $C_{1} \subseteq^{\varepsilon} C_{2}$ iff

$$
\sup \left\{\inf \left\{\|x-y\| \mid y \in C_{2},\|y\| \leq 1\right\} \mid x \in C_{1},\|x\| \leq 1\right\}<\varepsilon
$$

and

$$
d\left(C_{1}, C_{2}\right)=\inf \left\{\varepsilon>0 \mid C_{1} \subseteq^{\varepsilon} C_{2} \text { and } C_{2} \subseteq^{\varepsilon} C_{1}\right\}
$$

whenever $C_{1}, C_{2}$ are subspaces of $A$. If $\sigma$ is an almost inductive limit automorphism and $\varepsilon>0$, then there exists a unitary $v \in A$ such that $A d v \circ \sigma$ is an inductive limit automorphism and $\|v-1\|<\varepsilon$, [Voi, Proposition 2.3]. That $\beta=A d v \circ \sigma$ is an inductive limit automorphism means that there is an increasing sequence $\left(A_{n}\right) \subseteq \mathcal{F}(A)$ such that $\bigcup_{n} A_{n}$ is dense in $A$ and $\beta\left(A_{n}\right)=A_{n}$ for all $n$.

It is an open problem whether or not any automorphism of a UHF algebra is an almost inductive limit automorphism. If in particular $A=M_{2 \infty}=$ $\otimes_{2} M_{2}$, i.e. $A$ is the $C A R$ algebra, and $\sigma$ is the (Bernoulli) shift on $A$, it was established in [BKRS] that $\sigma$ has the Rohlin property, and hence $\sigma$ is an almost inductive limit automorphism. Let $\sigma$ be a quasifree automorphism of $A$, i.e.

$$
\sigma(a(f))=a(U f)
$$

where $f \in \mathcal{H}=$ the one particle space, $a(f)$ is the annihilator corresponding to $f$, and $U$ is a unitary operator on $\mathcal{H}$. It was established in [BEK] that $\sigma$ has the Rohlin property if and only if $U^{n}-1$ is not of Hilbert-Schmidt class for all $n \in \mathbb{Z} \backslash\{0\}$, i.e. if and only if $\sigma^{n}$ is outer in the trace representation for all $n \in \mathbb{Z} \backslash\{0\}$. Thus $\sigma$ is an almost inductive limit automorphism in this case. If on the other hand $U^{n}-1$ is a Hilbert-Schmidt operator for some $n \neq 0$, then $U$ has purely discrete spectrum and $\sigma$ is a product type automorphism, and hence $\sigma$ is a (strict) inductive limit automorphism.

The present paper came out of an attempt to establish that the shift $\sigma$ on $A=M_{n \infty}=\stackrel{\infty}{\otimes} M_{n}$ has the Rohlin property also for $n=3,5, \ldots$ (when $n$ is even, the Rohlin property follows from the Rohlin property of the shift on $M_{200}$ ). We have, however, only been able to establish the following approximate version of the Rohlin property.

Theorem 1. Let $\sigma$ be the one-sided shift on $M_{2^{\infty}}$, and let $\tau$ be the trace state on $M_{n \infty}$. For any finite subset $\left\{x_{1}, \ldots, x_{k}\right\} \subseteq M_{n^{\infty}}$, any $m \in \mathbf{N}$ and any $\varepsilon>0$ there exists projections $e_{0}, e_{1}, \ldots, e_{2^{m}}$ in $M_{n \infty}$ such that

$$
\begin{aligned}
& e_{2^{m}}=e_{0} \\
& e=\sum_{i=0}^{2^{m}-1} e_{i} \text { is a projection, }
\end{aligned}
$$




$$
\begin{aligned}
& \tau(e)>1-\varepsilon, \\
& \left\|\sigma\left(e_{i}\right)-e_{i+1}\right\|<\varepsilon
\end{aligned}
$$

for $i=0,1, \ldots, 2^{m}-1$, and

$$
\left\|\left[x_{j}, e_{i}\right]\right\|<\varepsilon
$$

for $i=0,1, \ldots, 2^{m}-1, j=1, \ldots, k$.

Combining Theorem 1 with the techniques of [Voi], [BKRS] we then establish that the shift is approximately an almost inductive limit automorphism in the following sense:

Corollary 2. Let $\sigma$ be the one-sided shift on $M_{n \infty}$. For any finite dimensional sub-*algebra $D$ of $M_{n \infty}$ and any $\varepsilon>0$, there exists a projection $e \in D^{\prime} \cap M_{n \infty}$ such that

$$
\|\sigma(e)-e\|<\varepsilon
$$

and

$$
\tau(e)>1-\varepsilon,
$$

and there exists a finite-dimensional ${ }^{*}$-subalgebra $E \subseteq e M_{n \infty} e$ such that

$$
D e \subseteq^{\varepsilon} E
$$

and

$$
d(E, \sigma(E))<\varepsilon
$$

The embedding of $\operatorname{GICAR}(\mathcal{H})$ into $M_{n} \infty$

In this section we prove Theorem 1 and Corollary 2. Let $\sigma$ be the one-sided shift on $M_{n \infty}, n=2,3,4, \ldots$ Let $\operatorname{GICAR}(\mathcal{H})$ be the gauge-invariant part of the $C A R$ algebra $C A R(\mathcal{H})$ over the Hilbert space $\mathcal{H}$, i.e., $\operatorname{GICAR}(\mathcal{H})$ consists of the elements in $C A R(\mathcal{H})$ which are invariant under the quasifree action of the circle group $T$ given by

$$
a(f) \rightarrow a(z f)
$$

for $z \in \mathbf{T} \subseteq \mathbf{C}, f \in \mathcal{H}$. Following [CE] (see also [E]), GICAR(H) can be embedded into $M_{n \infty}$ as follows: Let $\left(f_{i j}\right)_{i, j=1}^{n}$ be a complete set of matrix units for $M_{n}$, and define projections $e_{1}, e_{2}, \ldots$ in $M_{n \infty}=\bigotimes_{1}^{\infty} M_{n}$ as follows

$$
\begin{aligned}
& e_{1}=\sum_{i j} \frac{1}{n} f_{i j} \otimes 1 \otimes 1 \otimes \cdots, \\
& e_{2}=\sum_{i} f_{i i} \otimes f_{i i} \otimes 1 \otimes \cdots
\end{aligned}
$$




$$
\begin{aligned}
& e_{3}=\sum_{i j} \frac{1}{n} 1 \otimes f_{i j} \otimes 1 \otimes \cdots, \\
& e_{4}=\sum_{i} 1 \otimes f_{i i} \otimes f_{i i} \otimes \cdots,
\end{aligned}
$$

etc. The $e_{i}$ 's satisfy the Temperley-Lieb relations

$$
\begin{aligned}
& e_{i} e_{j}=e_{j} e_{i} \quad \text { if } \quad|i-j| \geq 2, \\
& e_{k} e_{k \pm 1} e_{k}=\frac{1}{n} e_{k} .
\end{aligned}
$$

Furthermore,

$$
\sigma\left(e_{k}\right)=e_{k+2}
$$

for $k=1,2, \ldots$

Let $f_{1}, f_{2}, \ldots$ be a basis for $\mathcal{H}$. The one-sided quasi-free shift is the injective morphism of $C A R(\mathcal{H})$ defined by

$$
\beta\left(a_{k}\right)=a_{k+1}
$$

where $a_{k}=a\left(f_{k}\right), k=1,2, \ldots$ The shift $\beta$ restricts to an injective morphism of $\operatorname{GICAR}(\mathcal{H})$ which we also denote by $\beta$.

The embedding $\gamma$ of $G I C A R(\mathcal{H})$ into $M_{n \infty}$ is given by

$$
\begin{aligned}
& \gamma\left(a_{i}^{*} a_{i}\right)=e_{2 i} \\
& \gamma\left(a_{i}^{*} a_{i+1}\right)=e_{2 i}\left(1-n e_{2 i+1}\right) e_{2 i+2},
\end{aligned}
$$

[CE]. We outline the argument from [CE]: First note that if $g l(\mathrm{~N})$ denotes the Lie algebra of complex $\infty \times \infty$ matrices such that only finitely many matrix elements are non-zero, then

$$
H=\left[H_{i j}\right] \in g l(\mathbb{N}) \rightarrow Q(H)=\sum_{i j} H_{i j} a_{i}^{*} a_{j}
$$

is a Lie algebra morphism, i.e.

$$
Q\left(\left[H, H^{\prime}\right]\right)=\left[Q(H), Q\left(H^{\prime}\right)\right]
$$

Since

$$
\left[e_{i, i+1}, e_{i+1, i+2}\right]=e_{i, i+2}
$$

etc., it follows that

$$
\left[a_{i}^{*} a_{i+1}, a_{i+1}^{*} a_{i+2}\right]=a_{i}^{*} a_{i+2}
$$

etc. It follows that the "algebra generated by $a_{i}^{*} a_{i}, a_{i}^{*} a_{i+1}$ contains all elements of the form $a_{i}^{*} a_{j}, i, j=1,2, \ldots$ Since any gauge invariant polynomial in $a_{i}, a_{j}^{*}, i, j=1, \ldots$ may be written as a polynomial in $a_{i}^{*} a_{j}$ it follows that 
We are now ready to construct the approximate Rohlin towers of Theorem 2. For economy of notation we identify $\operatorname{GICAR}(\mathcal{H})$ with its image in $M_{n_{\infty}}$.

If $\lambda, \mu \in \mathbf{T} \subseteq \mathbf{C}$, we may choose unit vectors $f, g \in \mathcal{H}$ such that $f, g$ are orthogonal, and

$$
U f \approx \lambda f, \quad U g \approx \mu g
$$

where $U$ is the isometry implementing the one-sided shift on $\mathcal{H}$. Then

$$
v=a^{*}(f) a(g)
$$

is a partial isometry with

$$
\sigma(v) \approx \lambda \bar{\mu} v
$$

Furthermore

$$
\begin{aligned}
v^{*} v & =a(g)^{*} a(f) a(f)^{*} a(g) \\
& =a(f) a(f)^{*} a(g)^{*} a(g) .
\end{aligned}
$$

Here $a(f) a(f)^{*}$ and $a(g)^{*} a(g)$ are commuting projections in different tensor factors (if $f, g$ are chosen suitably) of trace $\frac{n-1}{n}$ and $\frac{1}{n}$, respectively, so

$$
\tau\left(v^{*} v\right)=\frac{n-1}{n^{2}}
$$

Also, as

$$
\begin{aligned}
v^{*} v & =a(f)^{*} a(g) a(g)^{*} a(f) \\
& =a(f)^{*} a(f) a(g) a(g)^{*}
\end{aligned}
$$

and the projections $a(f)^{*} a(f), a(g) a(g)^{*}$ are orthogonal to $a(f) a(f)^{*}, a(g)^{*} a(g)$, respectively, the projection $v v^{*}$ is orthogonal to $v^{*} v$,

$$
v^{*} v v v^{*}=0=v v^{*} v^{*} v
$$

Hence the $C^{*}$-algebra $C^{*}(v)$ generated by $v$ is isomorphic to $M_{2}$, and if $1_{v}=v^{*} v+v v^{*}$ is the identity of this $C^{*}$-algebra, then

$$
\tau\left(1_{v}\right)=\frac{2(n-1)}{n^{2}}
$$

Also

$$
\left.\sigma\right|_{C^{*}(v)} \approx \operatorname{Ad}\left(\begin{array}{cc}
1 & 0 \\
0 & \lambda \bar{\mu}
\end{array}\right)
$$

Now, repeat the construction with $f_{2}, g_{2} \perp f, g$ and $\left(f_{2}, g_{2}\right)$ orthonormal, and cut down by $1-1_{v}=1-v v^{*}-v^{*} v$ to obtain a partial isometry $v_{2}$ with

$$
\tau\left(v_{2} v_{2}^{*}\right)=\tau\left(v_{2}^{*} v_{2}\right)=\frac{n-1}{n^{2}}\left(1-2 \frac{(n-1)}{n^{2}}\right)
$$


and

$$
\sigma\left(v_{2}\right) \approx \lambda \bar{\mu} v_{2}
$$

so

$$
\tau\left(1-v v^{*}-v^{*} v-v_{2} v_{2}^{*}-v_{2}^{*} v_{2}\right)=\left(1-2 \frac{(n-1)}{n^{2}}\right)^{2}
$$

Continuing in this manner, we may construct partial isometries $v_{1}=v, \ldots, v_{m}$ such that the projections $v_{1} v_{1}^{*}, v_{1}^{*} v_{1}, v_{2} v_{2}^{*}, v_{2}^{*} v_{2}, \ldots, v_{m} v_{m}^{*}, v_{m}^{*} v_{m}$ are all orthogonal, and

$$
\sigma\left(v_{k}\right) \approx \lambda \bar{\mu} v_{k}
$$

for all $k=1, \ldots, m$ and

$$
\tau\left(1-\sum_{k=1}^{m}\left(v_{k} v_{k}^{*}+v_{k}^{*} v_{k}\right)\right)=\left(1-2 \frac{n-1}{n^{2}}\right)^{m}
$$

Now, put

$$
u_{m}=v_{1}+v_{2}+\cdots+v_{m}
$$

then $u_{m}$ is a partial isometry such that $u_{m} u_{m}^{*} \perp u_{m}^{*} u_{m}, \sigma\left(u_{m}\right) \approx \lambda \bar{\mu} u_{m}$ and

$$
\tau\left(1-u_{m} u_{m}^{*}-u_{m}^{*} u_{m}\right)=\left(1-2 \frac{n-1}{n^{2}}\right)^{m}
$$

Now repeating this construction, going further and further out in $\mathcal{H} \simeq l^{2}(\mathrm{~N})$ to find $f, g^{\prime}$ s and using $\frac{1}{2 \pi i} \log (\lambda \bar{\mu})=2^{-1}, 2^{-2}, 2^{-3}, \ldots, 2^{-m}$ (with the last $m$ being that of Theorem 1 ), one finds a set of matrix units $e_{i j}, i, j=1, \ldots, 2^{m}$, which may be taken to approximately commute with any finite set in $M_{n \infty}$, such that $\tau\left(\sum_{i=1}^{2^{m}} e_{i i}\right)>1-\varepsilon$ and

$$
\sigma\left(e_{i j}\right) \approx e_{i+1, j+1}
$$

where the addition of indices is modulo $2^{m}$ (see [BEK] for details). Putting $e_{i}=e_{i i}$, Theorem 1 follows.

The proof of Corollary 2 from Theorem 1 now follows the lines of the proofs of [Voi, Lemma 3.3] or [BKRS, Lemma 2.1]. By [Voi, Lemma 3.1], for $D \in \mathcal{F}\left(M_{n \infty}\right)$ and $m \in \mathbb{N}$ there are $B_{j} \in \mathcal{F}\left(M_{n \infty}\right), j=0,1, \ldots, m$ with

$$
\begin{aligned}
& B_{0}=B_{m}, \\
& d\left(\sigma\left(B_{i}\right), B_{i+1}\right)<\frac{5 \pi}{m}, \quad i=0, \ldots, m-1, \\
& D \subseteq B_{i}, \quad i=0,1, \ldots, m .
\end{aligned}
$$


If $e_{0}, \ldots, e_{m}$ is a Rohlin tower approximately commuting with $B_{0}, \ldots, B_{m}$ we may assume that $e=\sum_{k=0}^{m-1} e_{k}$ commute with $D$ by a small perturbation of $e$, and putting

$$
E \approx \sum_{i=0}^{m-1} B_{i} e_{i}
$$

$E$ has the desired properties.

\section{References}

[BEK] O. Bratteli, D. E. Evans and A. Kishimoto, The Rohlin property for quasifree automorphisms of the fermion algebra, under typing.

[BKRS] O. Bratteli, A. Kishimoto, M. Rørdam and E. Størmer, The crossed product of a UHF algebra by a shift, Preprint, July'92.

[CE] A. Connes and D. E. Evans, Embedding of $U(1)$-current algebras in noncommutative algebras of classical statistical mechanics, Commun. Math. Phys. 12 (1989), 507-525.

[Con] A. Connes, Outer conjugacy classes of automorphisms of factors, Ann. Scient. Ec. Norm. Sup., \& série, 8 (1975), 383-420.

[E] D. E. Evans, $C^{*}$-algebraic methods in statistical mechanics and field theory, Inter. J. Modern Physics 4 (1990), 1069-1118.

[HO] R. H. Herman and A. Ocneanu, Stability for integer actions on UHF $C^{*}$-algebras, J. Funct. Anal. 59 (1984), 132-144.

[Ren] J. Renault, A Groupoid Approach to $C^{*}$-algebras, LNM 793, Springer Verlag 1980.

[Voi] D. Voiculescu, Almost inductive limit automorphisms and embeddings into AF-algebras, Ergod. Th. \& Dyman. Sys. 6 (1986), 475-484. 